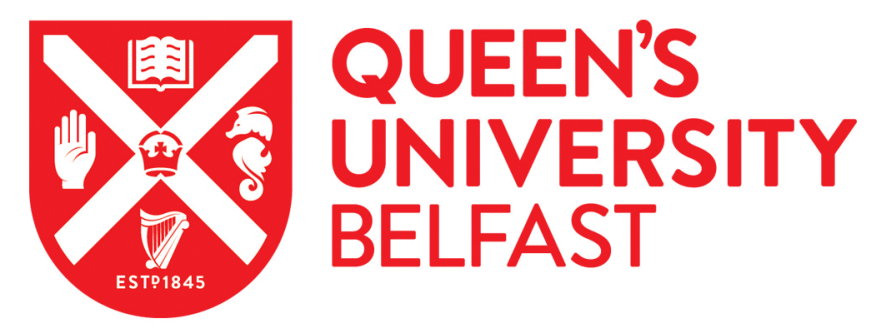

\title{
Exploring knowledge of pre-eclampsia and views on a potential screening test in women with type 1 diabetes
}

Wotherspoon, A., Young, I., McCance, D., \& Holmes, V. (2017). Exploring knowledge of pre-eclampsia and views on a potential screening test in women with type 1 diabetes. Midwifery.

https://doi.org/10.1016/j.midw.2017.03.019

\section{Published in:}

Midwifery

\section{Document Version:}

Peer reviewed version

\section{Queen's University Belfast - Research Portal:}

Link to publication record in Queen's University Belfast Research Portal

\section{Publisher rights}

Copyright 2017 Elsevier

This manuscript is distributed under a Creative Commons Attribution-NonCommercial-NoDerivs License

(https://creativecommons.org/licenses/by-nc-nd/4.0/), which permits distribution and reproduction for non-commercial purposes, provided the author and source are cited.

\section{General rights}

Copyright for the publications made accessible via the Queen's University Belfast Research Portal is retained by the author(s) and / or other copyright owners and it is a condition of accessing these publications that users recognise and abide by the legal requirements associated with these rights.

Take down policy

The Research Portal is Queen's institutional repository that provides access to Queen's research output. Every effort has been made to ensure that content in the Research Portal does not infringe any person's rights, or applicable UK laws. If you discover content in the Research Portal that you believe breaches copyright or violates any law, please contact openaccess@qub.ac.uk. 
Title: Exploring knowledge of pre-eclampsia and views on a potential screening test in women with type 1 diabetes

\section{Abstract}

\section{Objective}

To explore knowledge of pre-eclampsia and opinions on potential screening tests for pre-eclampsia in women with type 1 diabetes.

\section{Design}

A qualitative study using semi-structured interviews of women planning a pregnancy, currently pregnant or post-partum with experience of pre-eclampsia.

\section{Setting, Participants and Methods}

Eleven women with type 1 diabetes were recruited from a pre-pregnancy planning clinic or antenatal clinic. Semi-structured interviews were conducted with the women, asking a series of open-ended questions about their current knowledge of preeclampsia and their views on screening for pre-eclampsia. Data analysis was conducted using inductive thematic analysis.

\section{Findings}

Four main themes were identified: Information, sources of stress, awareness and acceptability of screening. Generally, women's knowledge of pre-eclampsia was limited. Most did not appear to be aware of their increased risk of developing the disease. Similarly, the majority of women were unaware as to why their blood pressure and urine were checked regularly. The introduction of a screening test for 
pre-eclampsia was favoured, with only a small number of women raising concerns related to the screening tests.

\section{Conclusions}

Health care professionals need to raise awareness of pre-eclampsia in this high risk group. The introduction of a screening test for pre-eclampsia appears to be acceptable in this population, however, further research is required to validate these findings and also to explore the views of women in other high risk groups.

\section{Keywords}

Diabetes, pre-gestational, pregnancy, information, pre-eclampsia, screening

Word count: 5,493; Abstract: 232 


\section{Introduction}

Pre-eclampsia is a hypertensive disorder of pregnancy defined as new-onset hypertension accompanied by proteinuria, maternal organ dysfunction or foetal growth restriction after 20 weeks gestation (Tranquilli et al., 2014). It is associated with significant neonatal and maternal morbidity and mortality (Backes et al., 2011). Although currently the only effective treatment for pre-eclampsia is delivery, low-dose aspirin started in early pregnancy ( 12-16 weeks) has been shown to reduce the risk of pre-eclampsia in women who are considered at high risk (Duley et al., 2007).

During pregnancy, maternal characteristics are reviewed to assess the risk of preeclampsia. Risk factors included age more 40 years, nulliparity, BMI greater than 30 , history of pre-eclampsia and diabetes (English et al., 2015). In addition blood pressure and urinary protein levels are monitored regularly for signs of pre-eclampsia (NICE, 2016a). While recent National Institute for Health and Care Excellence (NICE) guidelines advocate the use of assays measuring angiogenic markers, such as Placental Growth Factor (PIGF) and soluble fms-like tyrosine kinase-1 (sFlt-1) ratios, to 'rule-out' pre-eclampsia in all women suspected of having the condition (NICE, $2016 b)$, there is currently no effective screening test to predict pre-eclampsia in clinical practice.

Much research continues to focus on identification of accurate methods of predicting pre-eclampsia. Potential methods include the use of biochemical markers in blood and urine, biophysical measures, such as velocity indices obtained from ultrasound scans, and various combinations of these (O'Gorman et al., 2016; Poon and Nicolaides, 2014). However, very little research has explored the views of women on the acceptability of such tests. A study by Harris and colleagues ( 2014) assessed 
the psychological effect of providing women with risk information for pre-eclampsia. The study reported that women perceived there was an advantage to knowing the risk, despite the lack of effective treatment. Women felt that this information allowed them to be more prepared and may even help them to recognise the symptoms earlier. However, it is likely that women will react differently to the outcome of a screening test due to their own perception of risk, which is not entirely based on medical diagnosis (Heaman et al., 2004). How women view their pregnancy will be affected by their perception of risk (Lee et al., 2014), with women labelled as highrisk being negatively affected psychosocially (Stahl and Hundley, 2003). It is of particular interest to determine if women with diabetes, who are already considered high-risk, feel that an additional test for risk is acceptable or if this leads to medicalisation of pregnancy as previously suggested (Harris et al., 2014).To date, there have been no studies which have explored the views of women on screening for pre-eclampsia prior to introduction of a screening test. In addition to this, the views of women with type 1 diabetes, who are at substantially increased risk of preeclampsia, have not been explored. Therefore, the aim of this study was to provide insight into the knowledge of pre-eclampsia and views on implementation of a potential screening test for the condition in women with type 1 diabetes.

\section{Methods}

\section{Participants}

Ethical approval was granted from the South-West Exeter Research Ethics Committee (REC Ref: 14/SW/1015) prior to commencement of the study. Participants were recruited via linkage with the specialist pre-pregnancy care clinic or the joint antenatal-metabolic clinic within the Belfast Health and Social Care trust 
(BHSCT). Women were invited to participate if they were planning a pregnancy (nulliparous women only), currently pregnant or if they were up to 1 year postpartum. Women were eligible if they were aged 18 years or over and had a diagnosis of pre-gestational type 1 diabetes. Exclusion criteria included poor understanding of written or spoken English due to limited resources to facilitate translation and serious adverse outcome in a previous pregnancy (i.e. malformation or stillbirth), as discussion around this topic may have been upsetting.

\section{Procedure}

All potential participants were identified and approached by a healthcare professional and given an information sheet and an invitation slip which they returned to the researcher if they were happy to be contacted. Post-natal women were mailed the invitation/permission slip and the information sheet, alongside a stamped addressed envelope. At least 48 hours elapsed between the participant giving consent and the researcher contacting them to discuss the study. Interviews were scheduled for a time and place that suited the participant. Interviews lasted approximately 30-45 minutes, which included completion of consent forms, a background questionnaire and the interview. Participants were informed that interview would be audio recorded to allow for analysis to be conducted afterwards. Semi-structured interviews were conducted to allow the participants to introduce their own themes and ideas in relation to the topic, whilst ensuring the discussion remained relevant. Topic guides were used to guide the interviews and covered three main areas: advice for planning for pregnancy, knowledge about pre-eclampsia and screening for pre-eclampsia. For example, in relation to screening, women were asked 'Currently, risks factors are the only way of assessing what risk you have of developing pre-eclampsia. What would your opinion be on the introduction of a screening test for pre-eclampsia? This test 
may involve a blood test in combination with a more detailed scan and the risk factors that are currently used. The test could be delivered around 13 weeks (close to your current date) or 24 weeks'. It was deemed that women would have varying levels of knowledge in relation to pre-eclampsia so, if needed, an information leaflet from Action on Pre-eclampsia (Action on Pre-eclampsia, 2015) was discussed with women prior to discussion around screening for pre-eclampsia, allowing for more relevant discussion.

\section{Data analysis}

Interview recordings were transcribed verbatim and analysed through inductive thematic analysis, as described by Braun and Clarke, 2006 (Braun and Clarke, 2006). Six stages are described and are presented in Table 1. All transcripts were first read and re-read by A.C.W., with any comments/statements of interest being underlined to allow development of preliminary coding ideas. Transcripts were then reviewed and coded systematically. Codes and statements that were related to each code were extracted and tabulated, with similar codes being grouped into themes. Themes were reviewed and divided into sub-themes if needed. Themes and subthemes were discussed with a second researcher (V.A.H.) to ensure they reflected the data. 
Table 1: Phases of thematic analysis as discussed by Braun and Clarke, 2006 (Braun and Clarke, 2006)

\begin{tabular}{|c|c|}
\hline Phase & Description of process \\
\hline 1. Familiarizing yourself with the data & $\begin{array}{l}\text { Transcribe the data, reading and re-reading } \\
\text { the data, noting down initial ideas }\end{array}$ \\
\hline 2. Generating initial codes & $\begin{array}{l}\text { Coding interesting features of the data in a } \\
\text { systematic fashion across the entire data set, } \\
\text { collating data relevant to each code }\end{array}$ \\
\hline 3. Searching for themes & $\begin{array}{l}\text { Collating codes into potential themes, } \\
\text { gathering all data relevant to each theme }\end{array}$ \\
\hline 4. Reviewing themes & $\begin{array}{l}\text { Checking if the themes work in relation to } \\
\text { coded extracts (level 1) and the entire data } \\
\text { set (level 2), generating a thematic map of the } \\
\text { analysis }\end{array}$ \\
\hline 5. Defining and names themes & $\begin{array}{l}\text { Ongoing analysis to refine the specifics of } \\
\text { each theme, and the overall story the analysis } \\
\text { tells, generating clear definitions and names } \\
\text { for each theme }\end{array}$ \\
\hline 6. Producing the report & $\begin{array}{l}\text { The final opportunity for analysis. Selection of } \\
\text { vivid, compelling examples, final analysis of } \\
\text { selected extracts, relating back to the analysis } \\
\text { of the research question and literature, } \\
\text { producing a scholarly report of the analysis }\end{array}$ \\
\hline
\end{tabular}

\section{Results}

\section{Demographics}

A total of twenty-one women were invited to participate in the study, with thirteen agreeing to participate. Of the nine women that were invited but did not participate, eight did not respond when contacted by the researcher and one woman stated a lack of time. Two of the thirteen women were excluded (due to adverse incidents in a previous pregnancy), thus eleven women participated. Table 2 shows characteristics of participants. Of the eleven women that participated, two were planning a pregnancy and nine were currently pregnant. Of women who were currently 
pregnant, mean (SD) gestational age was 24.6 (8.8) weeks. No post-partum women who had experienced pre-eclampsia were recruited to the study.

Table 2: Demographic information of participants

\begin{tabular}{lc}
\hline Characteristic & Mean $(\mathbf{S D})(\mathbf{n}=\mathbf{1 1})$ \\
\hline Status & \\
Planning pregnancy & 2 \\
Currently pregnant & 9 \\
Post-partum & 0 \\
Maternal age (years) & $30.2(5.4)$ \\
BMI (kg/m²) & $28.6(3.7)$ \\
Years in full time education & $16.3(3.0)$ \\
Diabetes duration & $17.3(11.1)$ \\
Ethnicity & \\
White & $11(100 \%)$ \\
Marital status & \\
Never married & $2(18 \%)$ \\
Married & $7(64 \%)$ \\
Co-habit & $2(18 \%)$ \\
Current smoker & $2(18 \%)$ \\
Nulliparous & $10(91 \%)$ \\
Planned pregnancy & $7(64 \%)$ \\
Received pre-pregnancy counselling & $7(64 \%)$ \\
\hline Data prepted mean $($ SD) and $(\%)$ &
\end{tabular}

Data are presented as mean (SD) and $\mathrm{n}(\%)$

\section{Themes}

Four main themes were identified from the thematic analysis: Women's reflection on information received, sources of stress, women's self-awareness of complications in pregnancy and factors affecting acceptability of screening. A thematic map was developed to demonstrate themes and sub-themes that emerged from the data (Figure 1). 


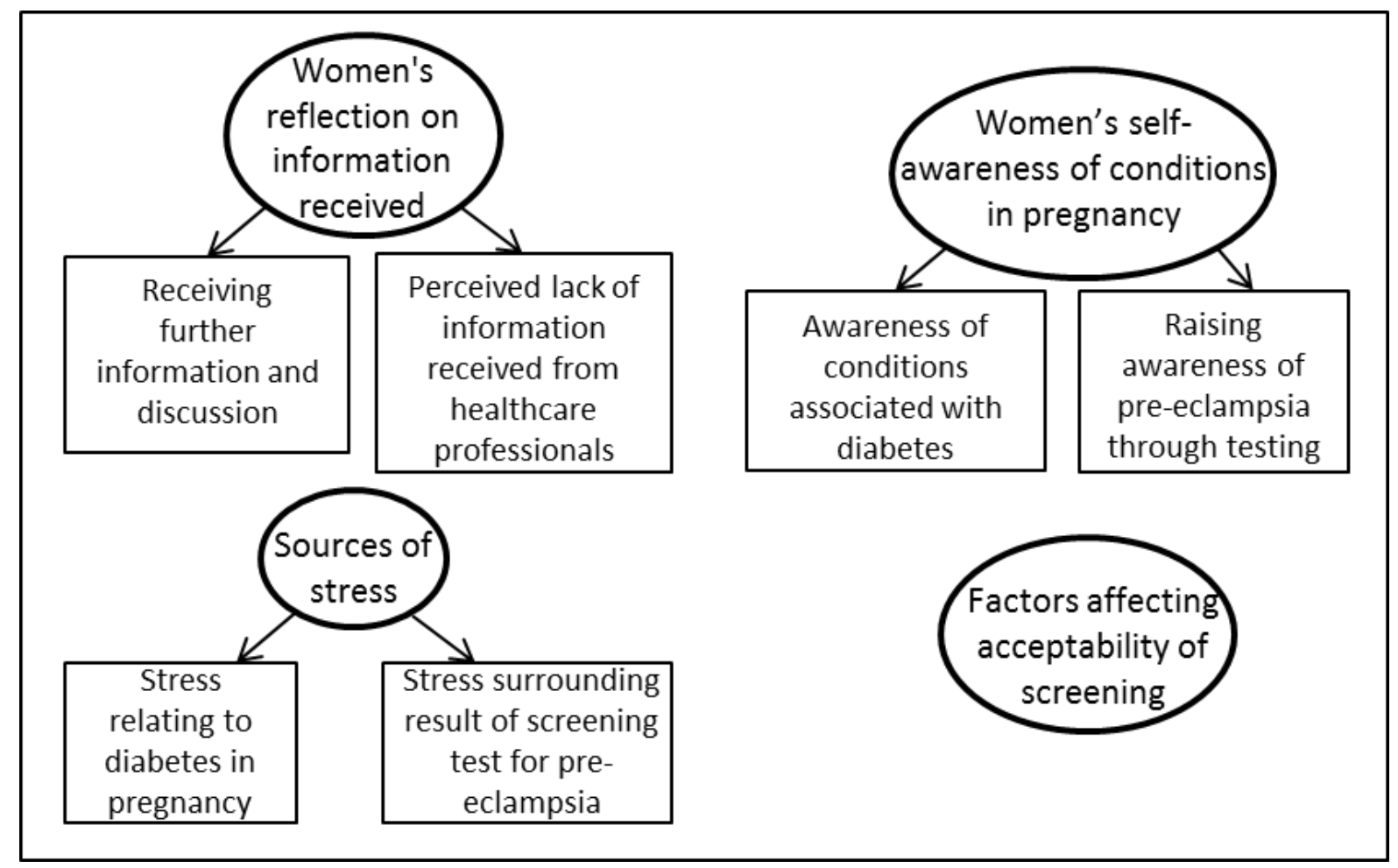

Figure 1: Thematic map of themes and sub-themes emerging from the data

\section{Women's reflection on information received}

The first theme consisted of two subthemes: Receiving further information and discussion and perceived lack of information received from healthcare professionals.

Receiving further information and discussion

Women generally stated that more information about conditions during pregnancy would be helpful, particularly if there were any risks involved, as is the case with preeclampsia. Women often explained that they had sought further information from external sources, including the internet, books or friends:

'Well if there were any risks, yea I would like to know. I would prefer to be more aware of signs cause I have never heard of it to be 
honest...if there were any risks l'd like to know.' - Participant 001, currently pregnant

‘ got a few pregnancy books and there was just a section on complications and pre-eclampsia was one of them.' - Participant 011, currently pregnant

One woman discussed how she felt about her appointments and believed that she did not have adequate time to ask questions as appointments were rushed, although she did appreciate that this was difficult as the clinic was very busy. She also stated that she liked to be involved in decisions about her care and would have preferred to discuss things further with or receive further information from her care team, particularly in relation to being prescribed aspirin:

'I like being involved you know? If you are going to be put on aspirin, I want to sort of talk about it, I don't want to just go...I'm not one of these people who just go ok and just go on it. I'll be like ok why and what's it going to do...because they obviously have a reason to put you on it but...l'm probably not looking in the right places...there is probably something out there that supports it so it would be good yea. But it probably comes down to time.' - Participant 008 , currently pregnant

\section{Perceived lack of information received from healthcare professionals}

Women appeared to receive varying levels of information, although most women said they were well supported by their diabetes care team and felt well prepared for their pregnancy. It became apparent that information received by women both prior to and 
during their pregnancy mainly centred on blood glucose targets and how this might affect their baby. Information around changing doses of insulin and monitoring blood sugars more frequently was also discussed. Most women did not recall receiving much other information:

'The only thing I received, because I was diabetic, was about blood sugar level control and that was pretty much it. That was really what it was focused around.' - Participant 009, currently pregnant

‘...but it was mainly just for bloods and how was your control. There was never really anything else.' - Participant 013, currently pregnant

Despite this, specific outcomes in relation to glycaemic control did not appear to be discussed. Women were asked if they received information about good glycaemic control reducing the risk of pre-eclampsia. The majority of women did not know this, but some did state that they assumed good control would be better overall:

'Like with a lot of things, they'll say oh because you've got diabetes, you're at high risk of this and higher risk of that but they don't really tell you that if your blood sugar...your HbA1c is very controlled the risk really drops...' - Participant 004, currently pregnant

A number of women also stated that at their booking appointment, they were given leaflets or a 'Pregnancy book', which contained information about various conditions, but this was never verbally discussed with them. Additionally, women highlighted that timing of information may be important. Some stated that they received a lot of information at their booking appointment, and that it may be more helpful to receive it further into their pregnancy: 
'You were just handed the book and it was kind of like it's in there, read it if you want, but I was never verbally told about it.' - Participant 013, currently pregnant 'yea, probably but then I think at your booking appointment you get told so much that it might be more beneficial to hear it a bit later on, a wee bit.' Participant 009, currently pregnant

Of women who were currently pregnant, only one woman recalled being told as to why her blood pressure and urine were being check regularly, with most being unaware as to why their blood pressure and urine was checked on a frequent basis:

'I obviously understood I was getting my blood pressure taken but I didn't know specifically what they were looking for.' - Participant 002, currently pregnant

'...he just told us that it had to be checked every so many weeks, every 6 weeks at least, but he didn't really go into detail why.' Participant 004, currently pregnant

In contrast, some women did discuss that they felt a little overwhelmed with information sometimes, and that there was a lot to take in so sometimes they may have forgotten what they were told:

'I got a lot of information so she probably has somewhere along the line...I think it's all the negative stuff sticks in your head and everything else just kind of goes over.' - Participant 004, currently pregnant 


\section{Sources of stress}

The second theme consisted of two sub-themes: stress relating to diabetes in pregnancy and stress surrounding result of pre-eclampsia screening test

Stress relating to diabetes in pregnancy

Stress related to a number of factors during pregnancy, ranging from discussing increased risks during pregnancy related to diabetes, to doubts about taking medications whilst pregnant, to how results of the potential screening test for preeclampsia would affect them.

Two women highlighted concern over taking tablets during pregnancy. As women with diabetes are considered high risk for pre-eclampsia, they are prescribed aspirin from 12 weeks onwards. These women recalled it being discussed and doubted if they should be going on medication during pregnancy:

'Yea, exactly! That was my main worry at the beginning, you taking another tablet on top of the folic acid and the pregnancy vitamins and stuff, you know, another tablet!' - Participant 002, currently pregnant

Women with diabetes are at an increased risk of a number of adverse outcomes and a number of women did recall being told this. However, this approach of being told the increased risk for multiple outcomes appeared to worry some women:

‘'m told I'm high risk for everything so l've pretty much just worried my entire pregnancy.' - Participant 011 , currently pregnant 
Stress surrounding result of screening test for pre-eclampsia

In relation to any future screening test for pre-eclampsia, it was suggested that the result of the test may cause potential stress. This issue was generally raised due to the lack of treatment available to women and so the value of knowing was questioned by some:

I'm sort of in two minds because as you say, there's not really a lot they can do with it once you have it so...in a way, it would be peace of mind to be checked but then once you have it, what can you do? It's not like they can give you a tablet to make it go away and as you say, the only cure is delivery. So what are you going to do? Sit for the last couple of weeks and worry?' - Participant 011 , currently pregnant

Although it was recognised that the result may be stressful, some women stated they would still prefer to know, even in terms of allowing them to take, what they deemed to be, helpful preventative measures:

‘ would still like to know! Don't get me wrong, I would be worried if there was more outcome of it but I would definitely like to know...it's just for yourself and the baby to keep safe. I know you can't do anything until the baby is delivered, but I would definitely like to know [...] just to see what they can do to help it or like myself with the diabetes, just to try and keep the bloods good...or maybe just do something that could help.' - Participant 001 , currently pregnant 
Others stated if the test result was low risk, it would still be in their minds but it would be less worrying:

'You probably wouldn't really consider it much, but I suppose if you had already been for that test, it would still always be in your mind, so if you did start having symptoms, you'd probably automatically think of it.' - Participant 009, currently pregnant

\section{Women's self-awareness of conditions in pregnancy}

Awareness consisted of two sub-themes: awareness of complications associated with diabetes and raising awareness of pre-eclampsia through testing.

Awareness of complications associated with diabetes

Women were very aware that having diabetes during pregnancy means that they were at high risk for a number of adverse outcomes and that pregnancy with diabetes was more complicated than a 'healthy' pregnancy:

'Well one, I'm diabetic so I knew there was going to be more complications involved...' - Participant 007, planning pregnancy

When asked if they were aware that having type 1 diabetes put them at an increased risk of pre-eclampsia, just over half of the women said that they were aware, which seemed to stem from when aspirin was prescribed to them:

'I think it was mentioned maybe 3 visits ago, just before the aspirin got prescribed to me. It was talked about then. I think that's when I 
first remember hearing the words pre-eclampsia.' - Participant 002, currently pregnant

With regard to awareness of signs and symptoms of pre-eclampsia, the response was mixed. Some women were able to list a number of symptoms, whilst others vaguely recalled a single symptom. Blood pressure was most often quoted as a sign, with only a small number of women knowing other symptoms like swelling and headaches. Sources of this kind of information also varied, and included healthcare professionals (i.e. obstetricians and general practitioner), friends and the internet:

'I know it's blood pressure. They check for blood pressure, check your urine for protein and then after that, I just assume headaches.'Participant 008, currently pregnant

Blood pressure, protein in your urine and swelling in your legs or...well particularly in your hands and face sometimes.' - Participant 010, currently pregnant

Raising awareness of pre-eclampsia through testing

In relation to screening for pre-eclampsia, women generally felt that screening would aid awareness of pre-eclampsia. It was perceived that the information received surrounding the screening test would allow women to be more aware of symptoms and to allow them to recognise them sooner:

'Yea, it maybe would then you could maybe...instead of waiting until your next appointment you could maybe phone somebody and get it checked out.' - Participant 008, currently pregnant 


\section{Factors affecting acceptability of screening}

Most women stated that if the screening test was offered to them that they would take it. The majority of women felt that it would be better to know your risk of preeclampsia, despite the fact that delivery is currently the only effective treatment:

'Yea but you still need to know...I think you're better off knowing really.' - Participant 007, planning pregnancy

I'd rather know. I prefer that to the unknown. I like to know about things, to know what to expect or something.' - Participant 009, currently pregnant

A number of factors determined the acceptability of the test, and women's opinions did differ in relation to these factors. The lack of treatment was mentioned as a limitation for some women, whilst others were unfazed about the lack of treatment:

[Iff] there was some preventative method or treatment...some option to delay things, then probably the screening would be more useful than whenever there isn't a preventative thing and the only treatment if having the baby.'- Participant 010 , currently pregnant

'Well I think I would want it either way. I would want to know and then...well if there is a treatment, I want the treatment.' - Participant 007, planning pregnancy

The method of testing also affected women's opinions. There was little issue over the test if it consisted of something non-invasive, such as an extra blood test or an extra ultrasound scan. However, opinion changed if there were risks to the baby associated with screening. As women attending the joint antenatal-metabolic clinic 
already have their blood taken every 2 weeks to monitor blood glucose control, they did not see an issue with someone taking additional blood to test it:

'Yea I would, I wouldn't at all. Obviously I am giving blood every 2 weeks anyway.' - Participant 004, currently pregnant 'No, well if you're going to be coming to appointments anyway and it's just a case of getting something simple like that done, then I don't really see that there should be any...like I wouldn't be saying no to it.' - Participant 013, currently pregnant

Accuracy and reliability were also discussed as important factors in deciding whether the test should be offered or if women would take it. One woman intimated she would be happy to take the test, even if it was not $100 \%$ accurate. Another woman stated that the test should only be offered if it was reliable. Having the option of taking the test was also raised by some women. It was felt that not all women would want to know their risk and so should be allow to refuse the test, and if they were interested in knowing, then they should be able to opt to have it:

'I think the option would be good to be there, in the same way in your 20 week scan you can get there anomaly check for that and then if they do find something, you can opt to have a deeper scan or not. I think that option should be left to you. I think definitely if it is offered but I think it should be left up to the mother if they want to take it or not.' - Participant 011, currently pregnant

In addition to this, most women were aware that the screening test may affect different women in different ways, highlighting that reaction would depend on 
personality. Women were generally very understanding of the notion that while they may not be concerned about result the test, others may be:

'...would cause some people a bit of extra stress. It just depends on how each person deals with it...'- Participant 005, planning pregnancy

I'm not much of a worrier, whereas some people, that might make them worry the whole...you know?' - Participant 009, currently pregnant

It was also highlighted that it might be better know your risk of pre-eclampsia in the earlier stages of pregnancy. One woman also went on to talk about the possibility of using this test result to stratify women based on their risk, and whether or not they received preventative measures (i.e. aspirin). Another woman added to this and felt that it may be more beneficial to only screen women who were in the higher risk categories:

'And the other thing is like all these preventative measures, but I don't think there is anything that says I am going to get it until I get it if you know what I mean? And maybe that would pick it up so you can have it quicker.' - Participant 008, currently pregnant

'I think if you were to fall into the higher risk group, something should be...if there is something to be offered, maybe it should be offered to the higher risk category...Makes more sense to monitor more the higher risk...'- Participant 013, currently pregnant 
Women who were currently pregnant were also asked how they would like the outcome to be presented to them if the test was introduced. Answers were mixed, with the majority of women saying either as a percentage or a high/low risk. One woman highlighted that the percentage would be stressful:

'If I got told I was $90 \%$ chance, then I would worry! I know I'm high risk but there's nothing screaming at you like you're 90\%, you're going to get this.' - Participant 008, currently pregnant

In contrast to this, another woman felt that high/low risk was too vague and that a percentage would be much more helpful:

'I like detail so I would probably go for the percentage, because I find it quite irritating as a diabetic just to be told...to be fired into a high risk category for everything.' - Participant 010 , currently pregnant Only two ladies opted for a visual representation of results. One lady said she would prefer the visual result and felt this would be much more helpful on the whole to other women:

'I don't think the format really matters but I think something visual over pages and pages of writing. Especially for people who maybe aren't as academically minded as others that visual things are always that wee bit easier to look at and understand...'- Participant 013, currently pregnant 


\section{Discussion}

\section{Summary}

This is the first study to investigate opinions of women surrounding potential screening for pre-eclampsia in women with type 1 diabetes. Generally, women were accepting of a potential screening test being introduced, although some women did raise concerns over the lack of effective prophylaxis and if the test posed a risk to their baby. The majority of women were also unaware as to why their blood pressure and urine were being measured on a regular basis and also stated that more information regarding pre-eclampsia would be helpful. However, number of women did express feelings of being overwhelmed with information during their pregnancy.

\section{Interpretation}

Research into opinions on screening for pre-eclampsia is scarce. A previous study (Harris et al., 2014) investigated the psychological impact of receiving risk information about pre-eclampsia in women were screened for pre-eclampsia and given a high or low-risk diagnosis. The study reported that most women regarded the screening test as acceptable and would like to be offered it in subsequent pregnancies. Some women did question the value of the test in light of the fact that there is no effect prophylaxis but also highlighted that they felt that would be better able to recognise the symptoms of pre-eclampsia if they received a high-risk diagnosis. The current study similarly found that women with type 1 diabetes, who are already at an increased risk of developing pre-eclampsia, stated that they thought a predictive test for pre-eclampsia would be useful. They also stated that the test would be helpful in terms of awareness of symptoms and what the condition was. These results are similar to those from a survey conducted by the Pre- 
eclampsia Foundation, asking women about their opinions of screening for preeclampsia (Preeclampsia Foundation, 2013). A total of 958 women responded, with $88 \%$ of women who had a history of pre-eclampsia saying that an early screening test would be beneficial, even if it was imperfect. The survey also found that $74 \%$ of women without a history of pre-eclampsia believed that the test would be beneficial. There was also a common consensus that risk information was important to know, even if there was no preventative intervention.

Women in the current study also stated that the test would be helpful in terms of awareness of symptoms and what the condition was, with many also stating that they were unaware of what pre-eclampsia was or had never heard of it. In contrast to this, further research from the Pre-eclampsia Foundation investigated the awareness of pre-eclampsia and found that although awareness was generally high, awareness of specific symptoms was low (Preeclampsia Foundation., 2014).Previous work by You and colleagues (2012b) suggested that women with pre-gestational diabetes had a better awareness of pre-eclampsia, as they were more likely to accurately define preeclampsia than those without pre-gestational diabetes. However, results from the current study would suggest that knowledge of pre-eclampsia amongst women with diabetes is low. Some women did mention that they received leaflets or the 'Pregnancy book' at their booking appointment. The 'Pregnancy book', that all women who are attending antenatal care should receive, contains information on signs and symptoms of pre-eclampsia (Department of Health., 2016). This may indicate that women simply did not read the information that they received which may highlight an issue surrounding the format of the information. A randomized controlled trial by You and colleagues (2012a) investigated the use of different formats of information for pre-eclampsia to improve patient knowledge of the condition. 
Participants were assigned to receive no intervention, a pamphlet from the American College of Obstetricians and Gynaecologists (ACOG) or the novel graphics-based tool. Women who received the graphics based tool demonstrated superior knowledge of pre-eclampsia compared to women who received the stand ACOG pamphlet or nothing. Additionally, women in the study reported here highlighted that timing of information may be important. Some women stated that they received a lot of information at their booking appointment, and that it may be more helpful to receive it later in pregnancy. It is therefore possible that women simply were unable to recall information due to the amount received at their booking appointment. Previous studies have shown that women with good blood glucose control, both prior to and during pregnancy, are at a reduced risk of pre-eclampsia, compared to those with poor glycaemic control (Cohen et al., 2014; Holmes et al., 2011). Despite this, the majority of women in the current study did not recall receiving information about the benefits of good glycaemic control on the risk of pre-eclampsia. This mirrors previous findings (Chuang et al., 2010; Spence et al., 2010) which found that women with diabetes, in particular nulliparous women, were aware that there are risks associated with diabetes in pregnancy but were not aware of specific risks. Additionally parous women with diabetes only reported knowing about the risk of a large baby and new-born glucose abnormalities. Also of note, women realised that importance of preconception care for management of their condition prior to pregnancy. It is therefore possible that women with diabetes are not being informed of specific risks (e.g. pre-eclampsia) in relation to their pregnancy by their healthcare professionals.

The majority of women in this study stated that they were unaware as to why their blood pressure and urine were being measured on a regular basis. This is despite 
the fact that current NICE guidelines for antenatal care state that women should be informed of the purpose of any test prior to it being performed (NICE, 2016a). However, a lack of information may relate to time pressures of staff within the department. The Patient Client Council (PCC) published a report in 2010, exploring patients' views of maternity services in Northern Ireland (Patient Client Council., 2010). The report found that patients felt that information (i.e. leaflets) was received at the first appointment without opportunity at a later stage to discuss the information or any concerns. Furthermore, patients cited that staff appeared to be under pressure and that more staff may improve the service received. This was similarly highlighted in the current study, with women stating they felt they did not have adequate time to discuss concerns or information and that their appointments were rushed.

\section{Strengths and weaknesses}

A major strength of this study was the format of semi-structured interviews for data collection. This approach allowed women to incorporate their own opinions and experiences whilst the conversation still remained relevant. Inclusion of both women who were currently pregnant and those who were planning their first pregnancy allowed any potential differences in knowledge due to stage of pregnancy to be highlighted, as women who were planning their pregnancy would not have received any information during a previous pregnancy. Of the women who were currently pregnant, a wide range of gestational ages were included (range 16-37 weeks), giving rich data across the childbirth trajectory. It was recognised that women would have varying knowledge of pre-eclampsia, so to enhance discussion surrounding screening for pre-eclampsia, the APEC leaflet was discussed with women after 
discussing their knowledge of pre-eclampsia and before discussion of screening tests.

There was difficulty in recruiting women who had experienced pre-eclampsia previously, due to the small numbers of women with type 1 diabetes attending antenatal care, and even smaller numbers of those who went on to develop preeclampsia. It was also only possible to recruit one woman who was multigravida, meaning that differences between information received across pregnancies could not be examined. Only women with type 1 diabetes were included, and may have differing opinions to women who are considered low risk during pregnancy and have less clinical contact. Additionally, only women of white ethnic origin participated and so views may not reflect women of other ethnic origins. Indeed, it has been highlighted that women from ethnic minorities are at higher risk of morbidity and mortality. It should also be noted that this study was only conducted on a single site and therefore may not represent views and opinions held in other areas.

\section{Conclusions}

Overall, women generally were accepting of the introduction of a potential screening test for pre-eclampsia. This is the first study to date to assess the opinions of women on the introduction of a screening test, prior to its implementation. As potential screening tests are currently being developed, it was important to explore the views of women about screening for pre-eclampsia, in light of the fact that there is no effective preventative treatment. The UK NSC currently does not recommend screening for pre-eclampsia, due to a lack of appropriate predictive tests or preventative treatment (United Kingdom National Screening Committee (UKNSC)., 2010). However, as research into more accurate predictive tests and preventative 
treatments continue (Dorniak-Wall et al., 2014; Duhig and Shennan, 2011; Kenny et al., 2010; O'Gorman et al., 2016), a screening test for pre-eclampsia may be introduced into clinical practice in the foreseeable future. It was therefore essential to explore the views of women around potential screening to assess the impact that it may have. Further research into this and other high risk maternal populations should be conducted to validate these findings and also highlight views of other women.

\section{References}

Action on Pre-eclampsia, 2015. Essential pregnancy information: why blood pressure and urine are measured during pregnancy. http://action-on-pre-eclampsia.org.uk/wpcontent/uploads/2012/07/Essential info leaflet 2015.pdf. 2016.

Backes, C.H., Markham, K., Moorehead, P., Cordero, L., Nankervis, C.A., Giannone, P.J., 2011. Maternal preeclampsia and neonatal outcomes. Journal of pregnancy 2011, 214365.

Braun, V., Clarke, V., 2006. Using thematic analysis in psychology. Qualitative research in psychology $3,77-101$.

Chuang, C.H., Velott, D.L., Weisman, C.S., 2010. Exploring knowledge and attitudes related to pregnancy and preconception health in women with chronic medical conditions. Maternal and child health journal 14, 713-9.

Cohen, A., Wenger, J., JAmes-Todd, T., Lamparello, B., Halprin, E., Serdy, S., Fan, S., Horowitz, G., Lim, K., Rana, S., Takoudes, T., Wyckoff, J., Thadhani, R., Karumanchi, S., Brown, F., 2014. The association of circulating angiogenic factors and $\mathrm{HbA} 1 \mathrm{c}$ with the risk of preeclampsia in women with preexisting diabetes. 33, 8192.

Department of Health., 2016. The pregnancy book: Problems in pregnancy. http://www.publichealth.hscni.net/sites/default/files/Chapter\%207\%20Conditions\%20 and\%20problems\%20in\%20pregnancy.pdf. Accessed 8/11 2016.

Dorniak-Wall, T., Grivell, R.M., Dekker, G.A., Hague, W., Dodd, J.M., 2014. The role of $L$-arginine in the prevention and treatment of pre-eclampsia: a systematic review of randomised trials. Journal of human hypertension 28, 230-5.

Duhig, K.E., Shennan, A.H., 2011. Antiplatelet agents for the prevention of preeclampsia. Seminars in thrombosis and hemostasis 37, 137-40.

Duley, L., Henderson-Smart, D., Meher, S., King, J., 2007. Antiplatelet agents for preventing pre-eclampsia and its complications. Cochrane Database Syst Rev 2. 
English, F.A., Kenny, L.C., McCarthy, F.P., 2015. Risk factors and effective management of preeclampsia. Integrated blood pressure control 8, 7-12.

Harris, J.M., Franck, L., Green, B., Michie, S., 2014. The psychological impact of providing women with risk information for pre-eclampsia: a qualitative study. Midwifery 30, 1187-95.

Heaman, M., Gupton, A., Gregory, D., 2004. Factors influencing pregnant women's perceptions of risk. MCN. The American journal of maternal child nursing 29, 111-6.

Holmes, V.A., Young, I.S., Patterson, C.C., Pearson, D.W., Walker, J.D., Maresh, M.J., McCance, D.R., 2011. Optimal glycemic control, pre-eclampsia, and gestational hypertension in women with type 1 diabetes in the diabetes and pre-eclampsia intervention trial. Diabetes care 34, 1683-8.

Kenny, L.C., Broadhurst, D.I., Dunn, W., Brown, M., North, R.A., McCowan, L., Roberts, C., Cooper, G.J., Kell, D.B., Baker, P.N., 2010. Robust early pregnancy prediction of later preeclampsia using metabolomic biomarkers. Hypertension 56, 741-9.

Lee, S., Ayers, S., Holden, D., 2014. A metasynthesis of risk perception in women with high risk pregnancies. Midwifery 30, 403-11.

NICE, 2016a. Antenatal Care for Uncomplicated Pregnancies. CG62.

NICE, 2016b. PIGF-based testing to help diagnose suspected pre-eclampsia (Triage PIGF test, Elecsys immunoassay sFlt-1/PIGF ratio, DELFIA Xpress PIGF 1-2-3 test, and BRAHMS sFlt-1 Kryptor/BRAHMS PIGF plus Kryptor PE ratio). DG23.

O'Gorman, N., Wright, D., Syngelaki, A., Akolekar, R., Wright, A., Poon, L.C., Nicolaides, K.H., 2016. Competing risks model in screening for preeclampsia by maternal factors and biomarkers at 11-13 weeks gestation. American Journal of Obstetrics and Gynecology 214, 103.e1,103.e12.

Patient Client Council., 2010. Parental Views on Maternity Services. http://www.patientclientcouncil.hscni.net/uploads/research/Strategic Review of Mat ernity Services.pdf. 2016.

Poon, L.C., Nicolaides, K.H., 2014. Early prediction of preeclampsia. Obstetrics and gynecology international 2014, 1-11.

Preeclampsia Foundation, 2013. Screening tests for preeclampsia. http://www.preeclampsia.org/the-news/3-newsflash/283-screening-tests-forpreeclampsia. Accessed February 25th 2014.

Preeclampsia Foundation., 2014. Pre-eclampsia Awareness Survey. https://www.preeclampsia.org/images/AwarenessMonth/Preeclampsia Study Result s Jan2014 FINAL.pdf. Accessed January 25th 2017. 
Spence, M., Alderdice, F., Harper, R., McCance, D., Holmes, V., 2010. An exploration of knowledge and attitudes related to pre-pregnancy care in women with diabetes. Diabetic Medicine 27, 1385-91.

Stahl, K., Hundley, V., 2003. Risk and risk assessment in pregnancy - do we scare because we care? Midwifery 19, 298-309.

Tranquilli, A.L., Dekker, G., Magee, L., Roberts, J., Sibai, B.M., Steyn, W., Zeeman, G.G., Brown, M.A., 2014. The classification, diagnosis and management of the hypertensive disorders of pregnancy: A revised statement from the ISSHP.

Pregnancy hypertension 4, 97-104.

United Kingdom National Screening Committee (UKNSC)., 2010. The UK NSC recommendation on Pre-eclampsia screening in pregnancy.

http://legacy.screening.nhs.uk/pre-eclampsia. 2016.

You, W.B., Wolf, M.S., Bailey, S.C., Grobman, W.A., 2012a. Improving patient understanding of preeclampsia: a randomized controlled trial. American Journal of Obstetrics and Gynecology 206, 431. e1,431. e5.

You, W.B., Wolf, M., Bailey, S.C., Pandit, A.U., Waite, K.R., Sobel, R.M., Grobman, W., 2012b. Factors associated with patient understanding of preeclampsia.

Hypertension in pregnancy $31,341-9$. 